\title{
Correlação entre achados manométricos e sintomatologia na incontinência fecal
}

\author{
Correlation between manometric findings and symptomatology \\ in fecal incontinence
}

\author{
FLÁVIA BALSAMO ${ }^{1}$, PAULO ROBERTO RAMACIOTTI FILHO², BÁRBARA HELOÍSA ZANCHETTA POZZOBON², \\ CARLOS ALBERTO TORRES DE CASTRO², GALDINO JOSÉ SITONIO FORMIGA ${ }^{3}$
}

\begin{abstract}
${ }^{1}$ Titular da Sociedade Brasileira de Coloproctologia; Assistente do Serviço de Coloproctologia do Hospital Heliópolis
- São Paulo (SP), Brasil. ${ }^{2}$ Filiados da da Sociedade Brasileira de Coloproctologia; Ex-residentes do Serviço de Coloproctologia do Hospital Heliópolis - São Paulo (SP), Brasil. ${ }^{3}$ Titular da Sociedade Brasileira de Coloproctologia; Chefe do Serviço de Coloproctologia do Hospital Heliópolis - São Paulo (SP), Brasil.
\end{abstract}

BALSAMO F, FILHO PRR, POZZOBONBHZ, CASTRO CAT, FORMIGAGJS. Correlação entre achados manométricos e sintomatologia na incontinência fecal. Rev bras Coloproct, 2011;31(1): 39-43.

RESUMO: Foram estudados 92 pacientes com sintomatologia de incontinência fecal, no período de julho de 2005 a fevereiro de 2009. Os sintomas de incontinência foram classificados de acordo com o Cleveland Clinic Incontinence Score e os pacientes foram submetidos à manometria anorretal. Foram avaliados: idade, sexo, cirurgia prévia, sintomatologia e achado manométrico. Observou-se que o aumento da intensidade da sintomatologia de incontinência fecal está relacionado à diminuição progressiva das pressões de repouso. O mesmo não foi constatado com as pressões de contração na amostra estudada.

Palavras-chave: incontinência fecal; manometria; complicações; canal anal; cirurgia.

\section{INTRODUÇÃO}

A incontinência fecal, definida como perda do controle voluntário das fezes ${ }^{1-4}$, é um grande problema social e de higiene. Afeta entre 1 e $15 \%$ dos adultos e 1,1 a $1,3 \%$ das pessoas com mais de 65 anos, impondo limitações e queda na qualidade de vida ${ }^{1,2}$. Sua real prevalência é provavelmente subestimada, já que a abordagem do sintoma é dificultada pelo constrangimento que tal afecção acarreta ${ }^{4-6}$.

Dentre os mais variados graus de incontinência fecal, o soiling (escape de material fecal pelo ânus após defecação normal, que pode levar à eczema perianal) ${ }^{7}$, quando incluso em questionários sobre incontinência, está presente em pelo menos 5\% dos indivíduos sadios e estes confirmam ter apresentado tal experiência em algum momento de suas vidas ${ }^{3}$.
A continência fecal é resultado da atividade coordenada entre o reto e os esfíncteres anais ${ }^{4}$ e depende não só destes, mas também da sensibilidade retal, tempo de trânsito intestinal, consistência das fezes e das condições do reservatório retal ${ }^{2,6-9}$.

Uma vez manifestada a incontinência fecal, sua severidade deve ser identificada. A incontinência leve consiste na perda involuntária de gases, perda ocasional de líquidos ou até eventualmente soiling. Incontinência grave se apresenta como a perda significante de líquidos ou sólidos e a necessidade do uso de forros $^{2,3}$.

Entretanto, classificar a incontinência não é tarefa fácil e enúmeros autores propuseram escalas e questionários para melhor graduar tal sintomatologia ${ }^{6,10}$.

Por vezes, não é possível estabelecer a etiologia da disfunção, sendo necessários métodos diagnósticos

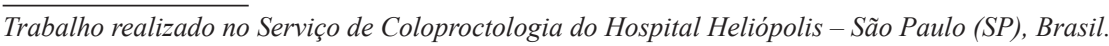


como manometria anorretal, tempo de latência do nervo pudendo, ultrassonografia endoanal, entre outros ${ }^{9,11}$.

A manometria anorretal é o exame de avaliação fisiológica mais largamente utilizado no estudo da incontinência fecal e permite a mensuração das pressões de repouso e contração, assim como o tamanho do canal anal funcional, capacidade, complacência e pesquisa do reflexo inibitório retoanal ${ }^{2}$, bem como entender a sincronização dos componentes sensórios e motores do canal anal'

A correlação dos dados manométricos com a gravidade dos sintomas de incontinência fecal e seu valor prognóstico foram objeto de muitos estudos na última década, apresentando, muitas vezes, resultados conflitantes ${ }^{6}$.

O objetivo deste trabalho é estudar a correlação entre achados pressóricos manométricos e a sintomatologia da incontinência fecal, principalmente quanto ao seu grau de intensidade e gravidade.

\section{MÉTODOS E PACIENTES}

Foi realizado estudo retrospectivo, no período de julho de 2005 a fevereiro de 2009, com 92 portadores de incontinência fecal, provenientes do ambulatório de coloproctologia do Hospital Heliópolis.

Foram resgatados dados de prontuários de ambulatório referentes à sintomatologia da incontinência fecal relatada. Tal sintomatologia foi classificada de acordo com o Cleveland Clinic Incontinence Score (Tabela 1).

Os pacientes foram submetidos à manometria anorretal pela técnica de perfusão de água, com equipamento Proctosystem de oito canais e cateter de perfusão radial. As medidas foram obtidas com intervalos de $1 \mathrm{~cm}$, com tração manual, iniciando-se no reto a 6 $\mathrm{cm}$ da margem anal, progredindo para o canal anal e foram mensuradas (em $\mathrm{mmHg}$ ) as pressões de repouso e contração, a capacidade e complacência retais e o reflexo inibitório retoanal.

Foram analisadas: idade, sexo, presença de cirurgia anorretal ou colorretal prévias, antecedentes obstétricos, bem como foram compilados os achados manométricos.

A sintomatologia relatada pelos pacientes foi confrontada com os achados manométricos e os dados foram submetidos à análise estatística, segundo os testes de qui-quadrado, comparação de médias ANOVA one-way e correlação de Pearson-Spearman com valores significantes de $\mathrm{p}<0,05$.

\section{RESULTADOS}

Foram estudados 92 portadores de incontinência fecal, no período de julho de 2005 a fevereiro de 2009.

A idade dos pacientes variou de 17 a 99 anos, com média de 59,6 anos. O sexo feminino foi predominante na amostra, correspondendo a 69 pacientes (75\%).

Quanto aos antecedentes, 17,4\% foram submetidos a cirurgias anorretoperineais e 7,6\% a cirurgias abdominais, com anastomoses colorretais baixas. Dentre as mulheres, 40 (57,9\%) apresentaram partos vaginais (Figura 1).

Não houve significância estatística em relação ao sexo $(p=0,11)$, aos antecedentes de cirurgia anal $(p=0,59)$ e ao parto vaginal $(p=0,67)$, mas houve significância em relação à presença de anastomose colorretal prévia com a sintomatologia de incontinência fecal $(p=0,001)$ (Tabela 2).

Segundo a classificação Cleveland Clinic Incontinence Score, 29,3\% dos pacientes apresentavam incontinência leve; $65,2 \%$, moderada e $5,4 \%$, grave (Figura 2).

Tabela 1. Cleveland Clinic Incontinence Score ${ }^{10}$.

\begin{tabular}{lccccc}
\hline Tipo de incontinência & Nunca & Raramente & Ocasionalmente & Frequentemente & Sempre \\
\hline Sólidos & 0 & 1 & 2 & 3 & 4 \\
Líquidos & 0 & 1 & 2 & 3 & 4 \\
Gases & 0 & 1 & 2 & 3 & 4 \\
Uso de forro & 0 & 1 & 2 & 3 & 4 \\
Alteração do estilo de vida & 0 & 1 & 2 & 3 & 4 \\
\hline
\end{tabular}

Índice de incontinência: somatória dos pontos; Escore: 0-continência perfeita; 1-7-incontinência leve; 8-14-incontinência moderada e 15-20-incontinência grave. 


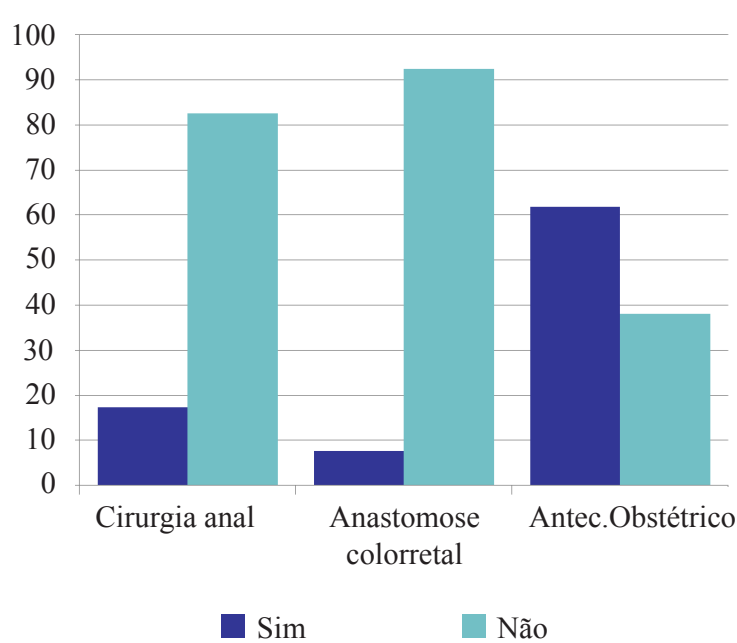

Figura 1. Antecedentes obstétricos e cirúrgicos.

Tabela 2. Relação entre anastomose colorretal prévia e sintomatologia de incontinência fecal.

\begin{tabular}{lcccc}
\hline Anastomose & \multicolumn{3}{c}{ Incontinência } & Total \\
\cline { 1 - 4 } Não & Leve & Moderada & Grave & \\
\cline { 2 - 5 } Sim & 27 & 56 & 2 & 85 \\
& $31,80 \%$ & $65,90 \%$ & $2,40 \%$ & $100 \%$ \\
Total & 0 & 4 & 3 & 7 \\
& $0 \%$ & $57,10 \%$ & $42,90 \%$ & $100 \%$ \\
& 27 & 60 & 5 & $92 \%$ \\
\cline { 1 - 4 } & $29,30 \%$ & $65,20 \%$ & 5,45 & $100 \%$ \\
\hline
\end{tabular}

Pearson chi-square $p=0,001$.

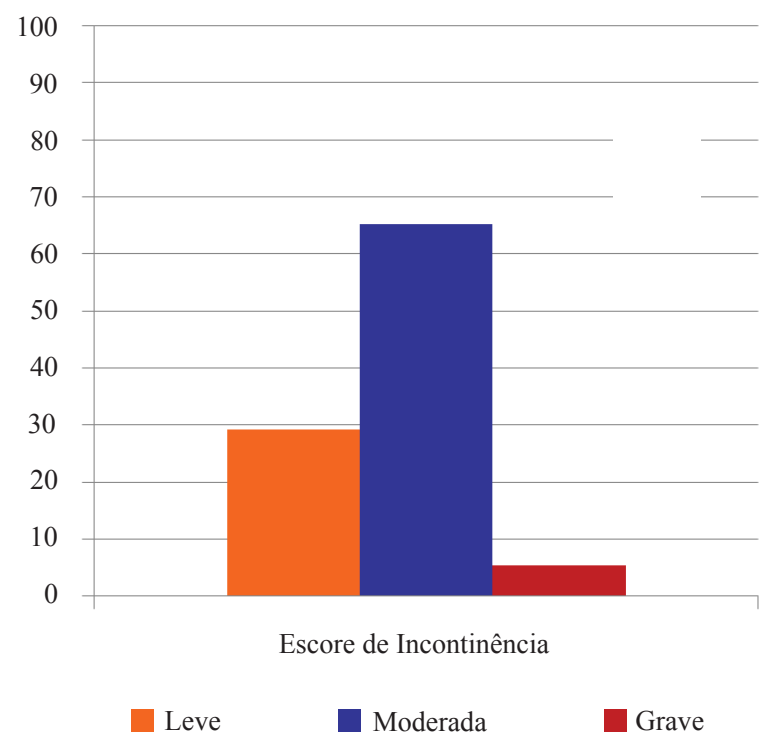

Figura 2. Classificação segundo o Cleveland Clinic Incontinence Score (CCIS).
Tabela 3. Correlação entre pressões de repouso e contração e a classificação CCIS.

\begin{tabular}{llccc}
\hline & & $\begin{array}{c}\text { Pressão } \\
\text { de } \\
\end{array}$ & CCIS & $\begin{array}{c}\text { Pressão } \\
\text { de }\end{array}$ \\
& & & & \\
Spearman's & Coeficiente & & & \\
ratio & de correlação & 1 & $-0,346$ & $-0,074$ \\
& Significância & & 0,001 & 0,482 \\
& $\mathrm{n}$ & 92 & 92 & 92 \\
\hline
\end{tabular}

Houve correlação entre a intensidade da sintomatologia de incontinência com a diminuição das pressões de repouso $(\mathrm{p}=0,001)$. Entretanto, não foi identificada tal correlação com as pressões de contração $(p=0,482)$, como pode ser visto na Tabela 3 .

Não houve significância estatística na relação entre os sintomas de incontinência fecal e os achados manométricos de sensibilidade retal, complacência, capacidade e pesquisa do reflexo inibitório retoanal.

\section{DISCUSSÃO}

Alguns estudos sugerem que a apresentação clínica de pacientes com incontinência fecal não é tão útil na identificação do mecanismo fisiopatológico que a origina ${ }^{7}$. Outros conseguiram identificar que a disfunção do esfíncter anal externo é o fator causal mais importante no aparecimento da urgência evacuatória, e a incontinência passiva ou soiling é de responsabilidade do esfíncter anal interno ${ }^{7}$.

Entretanto, diferentemente de outras síndromes gastrintestinais em que ocorre forte relação entre os sintomas e a doença, a incontinência fecal não apresenta tais relações, que não são fortes o suficiente para que sejam utilizadas exclusivamente na predição da anormalidade morfofuncional subjacente. Sendo assim, os testes funcionais são extremamente necessários nestes $\operatorname{casos}^{7}$. A associação entre a manometria anorretal, o tempo de latência do nervo pudendo, a eletroneuromiografia e a ultrassonografia endoanal fornecem nova informação diagnóstica em 53 a $98 \%$ dos casos e podem alterar o plano terapêutico em aproximadamente $20 \%$ dos pacientes ${ }^{12}$.

Tendo em vista estes achados, tentar estabelecer uma relação entre achados manométricos apenas e a sintomatologia da incontinência fecal torna-se um 
desafio, pois é de amplo conhecimento que a fisiologia da incontinência fecal ainda é pobremente compreendida ${ }^{8}$.

A classificação da gravidade da incontinência utilizada neste estudo (Cleveland Clinic Incontinence Score) não leva em conta a qualidade de vida, o que pode constituir crítica em relação ao seu uso. Foi escolhida e utilizada por sua simplicidade de execução e, principalmente, pelo fato dos dados terem sido provenientes da análise dos prontuários.

Os dados desta amostra são semelhantes à literatura, no correspondente à maior prevalência de incontinência no sexo feminino ${ }^{6}$ e que aumenta proporcionalmente com a idade.

O parto vaginal e a presença de cirurgias anorretais prévias e anastomoses colorretais baixas constituem fatores de risco ao aparecimento de incontinência $^{6}$, porém somente foi possível estabelecer relação entre a sintomatologia de incontinência fecal com a presença de anastomoses colorretais prévias. Entretanto, não há dados sobre a distância destas anastomoses em relação à margem anal.

Estudos demonstram que as pressões de repouso e contração são mais baixas em indivíduos incontinentes do que nos normais. Em relação à sintomatologia de incontinência, o mesmo já não ocorre ${ }^{6}$.

Neste estudo, foi evidenciado que houve correlação entre o aumento da intensidade e gravidade da sintomatologia de incontinência fecal com a diminuição das pressões de repouso, porém o mesmo não ocorreu em relação às pressões de contração. Isto pode ter ocorrido devido ao fato de ser uma amostra em que a maioria dos pacientes apresentava incontinência leve e moderada. Outros estudos não conseguiram estabelecer relação entre índice de incontinência e achados manométricos ${ }^{6}$.

Alguns autores analisaram pacientes com urgência evacuatória, soiling e combinação entre ambos e nenhuma relação foi identificada com a presença destes sintomas e a frequência dos episódios de incontinência. É provável que pacientes com tipos mais graves de incontinência tenham sintomatologia mais acentuada, tendo também maior comprometimento da função anorretal, bem como associação de outros mecanismos que comprometam a função anorretal, comparando-se com pacientes com incontinência mais leve?.

A correlação entre o escore de incontinência e os achados manométricos de mensuração da capacidade, complacência e reflexo inibitório retoanal não foram estatisticamente significantes, o que corresponde aos achados de outros estudos 6 .

\section{CONCLUSÃO}

A correlação entre os dados manométricos e a sintomatologia da incontinência fecal esteve relacionada principalmente aos parâmetros pressóricos e, neste quesito, a maior gravidade da incontinência fecal esteve diretamente relacionada com a diminuição das pressões de repouso, porém o mesmo não ocorreu em relação às pressões de contração na amostra estudada.

\footnotetext{
ABSTRACT: We have studied 92 patients with fecal incontinence symptoms, from July 2005 to February 2009. They were classified on the Cleveland Clinic Incontinence Score and all of them have undergone on manometric examination. Sex, age, previous surgery, symptomatology, and manometric findings were analyzed. We have concluded that the increase of incontinence symptoms is related to the progressive decrease on resting pressures. The same was not found with squeeze pressures in the studied sample.
}

Keywords: fecal incontinence; manometry; complications; anal canal; surgery.

\section{REFERÊNCIAS}

1. Norton C. Fecal Incontinence and biofeedback therapy. Gastroenterol Clin N Am 2008;37:587-603.

2. Hirsch T, Lembo T. Diagnosis and management of fecal incontinence in elderly patients. Am Fam Physician 1996;54:1559-64.
3. Jorge JMN, Wexner SD. Etiology and management of fecal incontinence. Dis Colon Rectum 1993;36:77-97.

4. Ness W. Faecal Incontinence: what influences care and management options? Br J Nurs 2008;17:1148-52.

5. Yusuf SAI, Jorge JMN, Habr-Gama A, Kiss DR, Rodrigues JG. Avaliação da qualidade de vida na incontinência anal: validação do questionário FIQL 
(Fecal Incontinence Quality of Life). Arq Gastroenterol 2004;41:202-8.

6. Carvalho LP, Corleta OC, Mallmann ACM, Koshimizu RT, Spolavori A. Neuropatia pudenda: correlação com dados demográficos, índice de gravidade e parâmetros pressóricos em pacientes com incontinência fecal. Arq Gastroenterol 2002;39:139-45.

7. Deutekom M, Dobben AC, Terra MP, Engel AF, Stoker J, Bossuyt PMM, et al. Clinical presentation of fecal incontinence and anorectal function: what is the relationship? Am J Gastroenterol 2007;102:351-61.

8. Ferrara A, Lujan JH, Cebrian J, Larach SW. Clinical, manometric and EMG characteristics of patients with fecal incontinence. Tech Coloproctol 2001;5:13-8.

9. Bhat NA, Grover VP, Bhatnagar V. Manometric evaluation of postoperative patients with anorectal anomalies. Indian J Gastroenterol 2004;23:206-8.

10. Oliveira L. Quality of life Issues. In: Complex anorectal disorders. USA: Springer. p. 595-605.

11. Viebig RG. Os desafios diagnósticos da incontinência fecal. Arq Gastroenterol 2002;39:137-8.

12. Wexner SD, Silva GM. Investigação funcional na incontinência fecal. In: Avaliação funcional em coloproctologia. USA: Reichmann \& Autores editores. p. $193-200$

\section{Endereço para correspondência:}

FLÁVIA BALSAMO

Serviço de Coloproctologia do Hospital Heliópolis

Rua Cônego Xavier, 276 - Vila Heliópolis

CEP: 04231-030 - São Paulo (SP), Brasil

E-mail: flabal@uol.com.br 